\title{
Single-photon signals at CERN LEP in supersymmetric models with a light gravitino
}

\author{
Jorge L. Lopez* \\ Bonner Nuclear Lab, Department of Physics, Rice University, 6100 Main Street, Houston, Texas 77005 \\ D. V. Nanopoulos \\ Center for Theoretical Physics, Department of Physics, Texas A\&M University, College Station, Texas 77843-4242 \\ and Astroparticle Physics Group, Houston Advanced Research Center (HARC), The Mitchell Campus, The Woodlands, Texas 77381 \\ A. Zichichi \\ University and INFN-Bologna, Italy \\ and CERN, 1211 Geneva 23, Switzerland \\ (Received 26 November 1996)
}

\begin{abstract}
We study the single-photon signals expected at CERN LEP in models with a very light gravitino $\left(m_{\widetilde{G}} \lesssim 10^{-3} \mathrm{eV}\right)$. The dominant process is neutralino-gravitino production $\left(e^{+} e^{-} \rightarrow \chi \widetilde{G}\right)$ with subsequent neutralino decay via $\chi \rightarrow \gamma \widetilde{G}$, giving a $\gamma+\mathrm{E}_{\text {miss }}$ signal. We first calculate the cross section at arbitrary center-ofmass energies and provide new analytic expressions for the differential cross section valid for general neutralino compositions. We then consider the constraints on the gravitino mass from LEP 1 and LEP 161 single-photon searches, and possible such searches at the Fermilab Tevatron. We show that it is possible to evade the stringent LEP 1 limits and still obtain an observable rate at LEP 2, in particular, in the region of parameter space that may explain the $\mathrm{CDF} e e \gamma \gamma+E_{T \text {,miss }}$ event. As diphoton events from neutralino pair production would not be kinematically accessible in this scenario, the observation of whichever photonic signal will discriminate among the various light-gravitino scenarios in the literature. We also perform a Monte Carlo simulation of the expected energy and angular distributions of the emitted photon, and of the missing invariant mass expected in the events. Finally we specialize the results to the case of a recently proposed one-parameter no-scale supergravity model. [S0556-2821(97)01809-2]
\end{abstract}

PACS number(s): 14.80.Ly, 12.60.Jv, $13.40 . \mathrm{Hq}$

\section{INTRODUCTION}

Supersymmetric models with a light gravitino $(\widetilde{G})$ have been considered for some time [1-5], but interest on them has recently surged [6-8] because of their ability to explain naturally the puzzling $e^{+} e^{-} \gamma \gamma+E_{T, \text { miss }}$ event observed by the Collider Detector at Fermilab (CDF) Collaboration [9]. If the gravitino is the lightest supersymmetric particle (LSP), ${ }^{1}$ the next-to-lightest supersymmetric particle [NLSP, typically the lightest neutralino $(\chi)$, as we will assume here] becomes unstable and eventually decays into a photon plus a gravitino $(\chi \rightarrow \gamma \widetilde{G})[2]$. This decay becomes of experimental interest when it happens quickly enough for the photon to be observed in the detector. Because the interaction of the gravitino with matter is inversely proportional to the gravitino mass, the neutralino lifetime will be short enough for a sufficiently light gravitino: $m_{\widetilde{G}} \lessgtr 250 \mathrm{eV}$ [7]. On the other hand, the gravitino may not be too light, as otherwise it would be copiously produced leading to distinctive signals at colliders that have not been observed [3,5] or cosmological [11] and astrophysical [12] embarrassments: $m_{\tilde{G}}>10^{-6} \mathrm{eV}$. Searches at the CERN $e^{+} e^{-}$collider LEP 1 strengthen this limit to $m_{\tilde{G}} \gtrsim 10^{-3} \mathrm{eV}$ in large regions of parameter space, when $m_{\chi}<M_{Z}[13]$.

\footnotetext{
*Permanent address: Shell E\&P Technology Company, 3737 Bellaire Boulevard, Houston, TX 77025.

${ }^{1}$ We assume that $R$ parity is conserved, as otherwise the decay $p \rightarrow \widetilde{G} K^{+}$may occur at an unsuppressed rate [10].
}

We should emphasize that even though we are encouraged by the natural interpretation of the CDF event within certain light-gravitino scenarios, we believe that such scenarios are interesting in their own right and should be fully explored irrespective of the status of the CDF event. This is the motivation for this analysis. One may parametrize models with very light gravitinos by the relation $m_{\widetilde{G}}$ $\sim\left(m_{1 / 2} / M_{\mathrm{Pl}}\right)^{p} M_{\mathrm{Pl}}$, with $m_{1 / 2}$ the gaugino mass scale and $p \sim 2$ a model-dependent constant. Specific forms of such mass relation have been obtained in the literature in the context of no-scale supergravity $[2,8]$. Light gravitinos are also expected in gauge-mediated models of low-energy supersymmetry $[6,14]$, where the gravitino mass is related to the scale of supersymmetry breaking via $m_{\tilde{G}} \approx 6 \times 10^{-5} \mathrm{eV}$ $\left(\Lambda_{\text {SUSY }} / 500 \mathrm{GeV}\right)^{2}$. However, the presently known models in this category appear unable to accomodate gravitino masses light enough to yield observable single-photon signals. Our analysis of direct experimental limits on the gravitino mass also complements analyses of indirect constraints on $m_{\widetilde{G}}$ from, e.g., cosmological [11] and astrophysical considerations [12].

Experimental searches for supersymmetry are considerably more sensitive in this type of neutralino-unstable supersymmetric model. First of all, the lightest easily observable supersymmetric channel is no longer a pair of charginos $\left(\chi^{+} \chi^{-}\right)$, but instead a pair of (the usually lighter) neutralinos $(\chi \chi)$, or if the gravitino is light enough $\left(m_{\widetilde{G}} \lesssim 10^{-3} \mathrm{eV}\right)$ the neutralino-gravitino channel $(\chi \widetilde{G})$. These new channels allow a deeper exploration into parameter space. Furthermore, because of the photonic signature in all supersymmetric processes, it becomes possible to over- 
come the loss of experimental sensitivity that occurs when the daughter leptons become too soft (as in chargino pair production when $m_{\chi^{ \pm}}-m_{\chi}<10 \mathrm{GeV}$ or $m_{\chi^{ \pm}}>m_{\widetilde{\nu}}>m_{\chi^{ \pm}}$ $-3 \mathrm{GeV}$ ), and therefore absolute lower bounds on sparticle masses become experimentally attainable in this class of models. Indeed, diphoton searches at LEP 161 (i.e., $\sqrt{s}=161 \mathrm{GeV}$ ) [15] have been recently shown [16] to exclude a significant fraction of the parameter space that is preferred by the supersymmetric interpretations of the CDF event within light gravitino models. ${ }^{2}$ Ongoing runs at LEP 2 should be able to probe even deeper into the remaining preferred region of parameter space.

Our purpose here is to consider in detail a complementary signal in light-gravitino models, namely the associated production of gravitinos with neutralinos, ${ }^{3}$ which may be observable in collider experiments for $m_{\tilde{G}} \lesssim 10^{-3} \mathrm{eV}$. The resulting single-photon signal has been recently shown to be observable at LEP 2 in certain range of gravitino masses, but only when the diphoton signal from neutralino pair production is itself not kinematically accessible [13]. Therefore, experimental observation of whichever photonic signal will provide very useful information in sorting out the various light-gravitino scenarios in the literature. The gravitino mass plays a central role in gravitino-production processes, whose rate is inversely proportional to the gravitino mass squared $\left(1 / m_{\widetilde{G}}^{2}\right)$. In contrast, the precise value of the gravitino mass plays a minor role in the production of the traditional supersymmetric particles, as it determines only the decay length of the neutralino. The neutralino-gravitino process of interest at LEP is

$$
\sigma\left(e^{+} e^{-} \rightarrow \chi \widetilde{G} \rightarrow \gamma+E_{\text {miss }}\right) \propto \frac{\beta^{8}}{m_{\widetilde{G}}^{2}}, \text { with } \beta=\sqrt{1-\frac{m_{\chi}^{2}}{s}},
$$

which provides an experimental handle on the gravitino mass. This process was considered originally by Fayet [3] (in the restricted case of a very light photinolike neutralino) who noted that the $\beta^{8}$ threshold behavior in Eq. (1) results from subtle cancellations among all contributing amplitudes. Dimensional analysis indicates that this cross section exceeds electroweak strength when $M_{Z}^{4} /\left(M_{\mathrm{Pl}}^{2} m_{\widetilde{G}}^{2}\right) \gtrless \alpha_{\text {weak }}$ or $m_{\widetilde{G}} \lesssim \alpha_{\text {weak }}^{-1 / 2} M_{Z}^{2} / M_{\mathrm{Pl}} \sim 10^{-4} \mathrm{eV}$. In the context of LEP 1, this process was reexamined in the restricted case of a neutralino with a non-negligible $Z$-ino component, where the resonant $Z$-exchange diagram dominates [5].

In this paper we first calculate the cross section for the $e^{+} e^{-} \rightarrow \chi \widetilde{G}$ process at arbitrary center-of-mass energies and

\footnotetext{
${ }^{2} \mathrm{We}$ should point out that alternative supersymmetric interpretations of the CDF event have been proposed, involving a one-loop radiative decay of the next-to-lightest neutralino [17] or a light axino [18]. Analyses of the impact of LEP searches on the allowed parameter spaces of these models have not yet appeared.

${ }^{3}$ Gravitino pair production $\left(e^{+} e^{-} \rightarrow \widetilde{G} \widetilde{G}\right)$ exceeds neutralinogravitino production $\left(e^{+} e^{-} \rightarrow \chi \widetilde{G}\right)$ only for gravitino masses that have already been excluded experimentally $\left(m_{\tilde{G}} \lessgtr 10^{-6} \mathrm{eV}\right)$. The single-photon signal is further suppressed by a factor of $\alpha$ from the radiated photon.
}

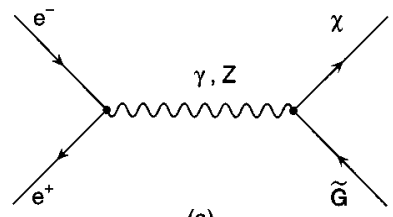

(a)

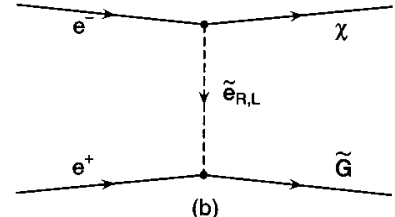

(b)
FIG. 1. Feynman diagrams for neutralino-gravitino production at LEP: (a) $s$-channel $\gamma$ and $Z$ exchange and (b) $t$-channel selectron exchange $\left(\widetilde{e_{R, L}}\right)$. Additional $u$-channel diagram not shown.

give new analytic expressions for the corresponding differential cross section (Sec. II). Next we reassess the constraints on the gravitino mass in view of the full LEP 1 data set and imposing the preliminary limits obtained recently from runs at LEP $161(\sqrt{s}=161 \mathrm{GeV})$, for general neutralino compositions (Sec. III). We also comment on the potential of analogous searches at the Tevatron. We then perform a Monte Carlo simulation of the production and decay processes leading to the single-photon signal and obtain energy $\left(E_{\gamma}\right)$ and angular $\left(\cos \theta_{\gamma}\right)$ distributions for representative points in parameter space (Sec. IV), and also discuss the missing invariant mass distribution expected in the events. We show that one may evade the LEP 1 limits and still obtain observable single-photon signals at LEP 2, although only when the diphoton signal from neutralino pair production is kinematically inaccessible. Finally we specialize our results to the case of our proposed one-parameter no-scale supergravity model $[8,19]$ (Sec. V). Our conclusions are summarized in Sec. VI.

\section{THE $e^{+} e^{-} \rightarrow \chi \widetilde{G}$ PROCESS}

The Feynman diagrams for neutralino-gravitino associated production at LEP are shown in Fig. 1, and include $s$-channel $\gamma$ and $Z$ exchange, and $t$ - and $u$-channel selectron $\left(\tilde{e}_{R, L}\right)$ exchange. At the $Z$ peak one expects the $s$-channel $Z$ exchange diagram to dominate, and one may simply calculate the amplitude for $Z \rightarrow \chi \widetilde{G}$ decay [5]. This result is accurate as long as the neutralino has a non-negligible $Z$-ino component. However, for photinolike neutralinos the other diagrams become important. This is also the case for any neutralino composition for center-of-mass energies away from the $Z$ peak $\left(\sqrt{s}>M_{Z}\right)$. To deal with all cases at once, we perform the complete calculation of all diagrams contributing to $e^{+} e^{-} \rightarrow \chi \widetilde{G}$. We first present the general form of the differential cross section and later specialize the result for the particular case of a photinolike neutralino in order to expound on certain theoretical issues and generalizations of our results.

In calculating interactions of gravitinos with matter one can proceed in one of two ways. One may calculate with the full couplings in the supergravity Lagrangian, in which case the vertices of interest are given by $[1,11]$

$e{\widetilde{e_{R, L}}}_{\widetilde{G}_{\mu}} \propto \frac{\gamma_{\nu} \gamma_{\mu}}{\sqrt{2} M} P_{R, L} p_{\widetilde{e}}^{\nu}, \quad \gamma_{\sigma} \widetilde{\gamma} \widetilde{G}_{\mu} \propto \frac{1}{M} p_{\gamma}^{\rho}\left[\gamma_{\rho}, \gamma_{\sigma}\right] \gamma_{\mu}$,

where the (spin- $\frac{1}{2}$ Goldstino component of the) gravitino field is $\widetilde{G}_{\mu} \propto \partial_{\mu} \psi / m_{\tilde{G}}$, and $M=2.4 \times 10^{18} \mathrm{GeV}$ is the appro- 
priately scaled Planck mass. Alternatively one may calculate using a set of much-simplified effective Goldstino couplings [3]

$$
e{\widetilde{e_{R, L}}} \psi \propto \frac{m_{\tilde{e}}^{2}-m_{e}^{2}}{\sqrt{6} M m_{\tilde{G}}} P_{R, L}, \quad \gamma_{\sigma} \tilde{\gamma} \psi \propto \frac{m_{\tilde{\gamma}}}{\sqrt{6} M m_{\tilde{G}}}\left[\gamma_{\rho}, \gamma_{\sigma}\right] p_{\gamma}^{\rho} .
$$

The full and effective couplings give the same results for the cross sections of processes where the typical bad high-energy behavior of the gravitational amplitudes is cancelled completely by the diagrams involving only gravitinos and regular supersymmetric particles. This is the case for the $e^{+} e^{-} \rightarrow \chi \widetilde{G}$ process in hand, where we have verified (in the pure photino limit) that both ways of doing the calculation give identical results. For a derivation and explanation of the meaning of the effective couplings see Ref. [11]. The simplification of using the effective couplings is not beneficial in other processes, such as gravitino pair production, where diagrams including graviton exchanges must also be included to cancel the bad high-energy behavior of the amplitudes $[3,11]$. Also, it is not clear whether this simplification may be used in the case of broken gauge symmetries, and therefore we have used the full couplings in the case of neutralino composit ions other than pure photino, where the $s$-channel $Z$-exchange amplitude must be taken into account.

\section{A. General case}

The differential cross section for a general neutralino composition is given by

$$
\frac{d \sigma}{d \cos \theta}=\frac{\left(s-m_{\chi}^{2}\right)}{32 \pi s^{2}} \frac{F(s, t, u)}{6\left(M m_{\tilde{G}}\right)^{2}},
$$

where as usual we define

$$
\begin{aligned}
& t=-\frac{1}{2}\left(s-m_{\chi}^{2}\right)(1-\cos \theta), \\
& u=-\frac{1}{2}\left(s-m_{\chi}^{2}\right)(1+\cos \theta) .
\end{aligned}
$$

The function $F(s, t, u)$ receives contributions from each amplitude squared and various interference terms (some of which vanish). In an obvious notation, we find

$$
F=F_{\gamma \gamma}+F_{t t}+F_{u u}+F_{z Z}+F_{\gamma t}+F_{\gamma u}+F_{Z t}+F_{Z u}+F_{\gamma Z},
$$

where

$$
\begin{gathered}
F_{\gamma \gamma}=\left(N_{11}^{\prime} e\right)^{2} \frac{2 s\left(s-m_{\chi}^{2}\right)\left(t^{2}+u^{2}\right)}{s^{2}} \\
F_{t t}=\left(X_{R}\right)^{2} \frac{t^{2}\left(m_{\chi}^{2}-t\right)(-t)}{\left(t-m_{\widetilde{e}_{R}}^{2}\right)^{2}}+\left(X_{L}\right)^{2} \frac{t^{2}\left(m_{\chi}^{2}-t\right)(-t)}{\left(t-m_{\widetilde{e}_{L}}^{2}\right)^{2}}, \\
F_{u u}=\left(X_{R}\right)^{2} \frac{u^{2}\left(m_{\chi}^{2}-u\right)(-u)}{\left(u-m_{\widetilde{e}_{R}}^{2}\right)^{2}}+\left(X_{L}\right)^{2} \frac{u^{2}\left(m_{\chi}^{2}-u\right)(-u)}{\left(u-m_{\widetilde{e}_{L}}^{2}\right)^{2}},
\end{gathered}
$$

$$
F_{Z Z}=\left(N_{12}^{\prime} \frac{g}{\cos \theta_{W}}\right)^{2} \frac{\left(c_{R}^{2}+c_{L}^{2}\right)}{2} \frac{2 s\left(s-m_{\chi}^{2}\right)\left(t^{2}+u^{2}\right)}{\left(s-M_{Z}^{2}\right)^{2}+\left(\Gamma_{Z} M_{Z}\right)^{2}},
$$

$F_{\gamma t}=\left(N_{11}^{\prime} e X_{R}\right) \frac{(-t)\left(2 s t^{2}\right)}{s\left(t-m_{\widetilde{e}_{R}}^{2}\right)}-\left(N_{11}^{\prime} e X_{L}\right) \frac{(-t)\left(2 s t^{2}\right)}{s\left(t-m_{\widetilde{e}_{L}}^{2}\right)}$,

$$
F_{\gamma u}=\left(N_{11}^{\prime} e X_{R}\right) \frac{(-u)\left(2 s u^{2}\right)}{s\left(u-m_{\widetilde{e}_{R}}^{2}\right)}-\left(N_{11}^{\prime} e X_{L}\right) \frac{(-u)\left(2 s u^{2}\right)}{s\left(u-m_{\widetilde{e}_{L}}^{2}\right)} \text {, }
$$

$$
\begin{aligned}
F_{Z t}= & -\left(N_{12}^{\prime} c_{R} X_{R} \frac{g}{\cos \theta_{W}}\right) \frac{(-t)\left(2 s t^{2}\right)\left(s-M_{Z}^{2}\right)}{\left[\left(s-M_{Z}^{2}\right)^{2}+\left(\Gamma_{Z} M_{Z}\right)^{2}\right]\left(t-m_{\widetilde{e}_{R}}^{2}\right)} \\
& +\left(N_{12}^{\prime} c_{L} X_{L} \frac{g}{\cos \theta_{W}}\right) \frac{(-t)\left(2 s t^{2}\right)\left(s-M_{Z}^{2}\right)}{\left[\left(s-M_{Z}^{2}\right)^{2}+\left(\Gamma_{Z} M_{Z}\right)^{2}\right]\left(t-m_{\widetilde{e}_{L}}^{2}\right)},
\end{aligned}
$$

$$
\begin{aligned}
F_{Z u}= & -\left(N_{12}^{\prime} c_{R} X_{R} \frac{g}{\cos \theta_{W}}\right) \frac{(-u)\left(2 s u^{2}\right)\left(s-M_{Z}^{2}\right)}{\left[\left(s-M_{Z}^{2}\right)^{2}+\left(\Gamma_{Z} M_{Z}\right)^{2}\right]\left(u-m_{\widetilde{e}_{R}}^{2}\right)} \\
& +\left(N_{12}^{\prime} c_{L} X_{L} \frac{g}{\cos \theta_{W}}\right) \frac{(-u)\left(2 s u^{2}\right)\left(s-M_{Z}^{2}\right)}{\left[\left(s-M_{Z}^{2}\right)^{2}+\left(\Gamma_{Z} M_{Z}\right)^{2}\right]\left(u-m_{\widetilde{e}_{L}}^{2}\right)},
\end{aligned}
$$

$$
\begin{aligned}
F_{\gamma Z}= & -2\left(N_{11}^{\prime} N_{12}^{\prime} e \frac{g}{\cos \theta_{W}}\right) \\
& \times \frac{\left(c_{R}+c_{L}\right)}{2} \frac{2 s\left(s-m_{\chi}^{2}\right)\left(t^{2}+u^{2}\right)\left(s-M_{Z}^{2}\right)}{s\left[\left(s-M_{Z}^{2}\right)^{2}+\left(\Gamma_{Z} M_{Z}\right)^{2}\right]} .
\end{aligned}
$$

In these expressions we have used the following $e-\widetilde{e}_{R, L^{-}} \chi$ $\left(X_{R, L}\right)$ and $e-e-Z\left(c_{R, L}\right)$ couplings [20]:

$$
\begin{gathered}
X_{R}=N_{11}^{\prime} e-N_{12}^{\prime} \frac{g \sin ^{2} \theta_{W}}{\cos \theta_{W}}, \\
X_{L}=-N_{11}^{\prime} e-N_{12}^{\prime} \frac{g}{\cos \theta_{W}}\left(\frac{1}{2}-\sin ^{2} \theta_{W}\right), \\
c_{R}=\sin ^{2} \theta_{W}, \\
c_{L}=-\frac{1}{2}+\sin ^{2} \theta_{W},
\end{gathered}
$$

where $N_{11}^{\prime}$ and $N_{12}^{\prime}$ denote the photino and $Z$-ino components of the neutralino, respectively. Indeed, the lightest neutralino may be written in two equivalent ways [20]:

$$
\begin{aligned}
& \chi=N_{11}^{\prime} \widetilde{\gamma}+N_{12}^{\prime} \widetilde{Z}+N_{13} \widetilde{H}_{1}^{0}+N_{14} \widetilde{H}_{2}^{0}, \\
& \chi=N_{11} \widetilde{B}+N_{12} \widetilde{W}_{3}+N_{13} \widetilde{H}_{1}^{0}+N_{14} \widetilde{H}_{2}^{0}
\end{aligned}
$$

related by 


$$
\begin{gathered}
N_{11}^{\prime}=N_{11} \cos \theta_{W}+N_{12} \sin \theta_{W}, \\
N_{12}^{\prime}=-N_{11} \sin \theta_{W}+N_{12} \cos \theta_{W} .
\end{gathered}
$$

\section{B. Pure photino case}

This special case is useful in order to expose various subtleties in the calculation that become less apparent (although they are still present) in the case of a general neutralino composition. The $e^{+} e^{-} \rightarrow \widetilde{\gamma} \widetilde{G}$ case is also important because the result can be readily taken over to the case of gluino-gravitino production in quark-antiquark annihilation at hadron colliders $(q \bar{q} \rightarrow \widetilde{g} \widetilde{G})$.

In this special case the couplings of the neutralino (photino: $\left.N_{11}^{\prime}=1, \quad N_{12}^{\prime}=0\right)$ to matter are very simple: $X_{R}=e=-X_{L}, F_{Z Z}=F_{Z t}=F_{Z u}=F_{\gamma Z}=0$, and

$$
F \rightarrow F^{\tilde{\gamma}}=e^{2}\left(F_{\gamma}^{\tilde{\gamma}}+F_{R}^{\tilde{\gamma}}+F_{L}^{\tilde{\gamma}}\right),
$$

where

$$
\begin{gathered}
F_{\gamma}^{\widetilde{\gamma}}=\frac{2 s\left(s-m_{\chi}^{2}\right)\left(t^{2}+u^{2}\right)}{s^{2}}, \\
F_{R}^{\widetilde{\gamma}}=\frac{t^{2}\left(m_{\chi}^{2}-t\right)(-t)}{\left(t-m_{\widetilde{e}_{R}}^{2}\right)^{2}}+\frac{u^{2}\left(m_{\chi}^{2}-u\right)(-u)}{\left(u-m_{\widetilde{e}_{R}}^{2}\right)^{2}} \\
+\frac{t^{2}(-2 s t)}{s\left(t-m_{\widetilde{e}_{R}}^{2}\right)}+\frac{u^{2}(-2 s u)}{s\left(u-m_{\widetilde{e}_{R}}^{2}\right)}, \\
F_{L}^{\tilde{\gamma}}=\frac{t^{2}\left(m_{\chi}^{2}-t\right)(-t)}{\left(t-m_{\widetilde{e}_{L}}^{2}\right)^{2}}+\frac{u^{2}\left(m_{\chi}^{2}-u\right)(-u)}{\left(u-m_{\widetilde{e}_{L}}^{2}\right)^{2}} \\
+\frac{t^{2}(-2 s t)}{s\left(t-m_{\widetilde{e}_{L}}^{2}\right)}+\frac{u^{2}(-2 s u)}{s\left(u-m_{\widetilde{e}_{L}}^{2}\right)} .
\end{gathered}
$$

With this relatively simple expression we can verify certain expected behaviors of the cross section. First, in the limit of unbroken supersymmetry $m_{\chi} \rightarrow m_{\gamma}=0, \quad m_{\tilde{e}_{L, R}}$ $\rightarrow m_{e} \approx 0, s+t+u=m_{\chi}^{2} \rightarrow 0$, one can readily verify from the above equations that $F^{\gamma} \rightarrow 0$. The vanishing of the cross section in this limit is expected as the spin- $\frac{1}{2}$ component of the gravitino (the Goldstino) becomes an unphysical particle when supersymmetry is unbroken, as it is no longer absorbed by the gravitino to become massive.

A related manifestation of this phenomenon can be exposed by studying the threshold behavior of the cross section. The spin- $\frac{1}{2}$ (Goldstino) component of the gravitino is essentially obtained by taking the derivative of the full gravitino field, thus making the Goldstino couplings proportional to the Goldstino momentum $\left(k^{\mu}\right)$. At threshold $k^{\mu} \rightarrow 0$ and there is an additional suppression of the cross section besides the kinematical one. Threshold corresponds to the limit $s \rightarrow m_{\chi}^{2}$ and therefore from Eqs. (5), (6) $t, u$ go to zero as $\left(s-m_{\chi}^{2}\right)$. In the above expression for $F^{\tilde{\gamma}}$, one can see that near threshold each term is proportional to $\left(s-m_{\chi}^{2}\right)^{3}$, which combined with the $\left(s-m_{\chi}^{2}\right)$ term from the phase space integration [see Eq. (4)] yields a cross section proportional to $\beta^{8}$ with $\beta=\sqrt{1-m_{\chi}^{2} / s}$.

The above results were originally obtained by Fayet [3] based on a calculation of the cross section using the effective couplings [Eq. (3)], and have been obtained here for the first time using the full couplings [Eq. (2)]. Such an equivalent expression for the cross section makes more evident some further properties of the results, and we thus give it explicitly here too. The expression for $F^{\widetilde{\gamma}}$ using the effective couplings becomes

$$
\begin{aligned}
F_{\mathrm{eff}}^{\tilde{\gamma}}= & m_{\chi}^{4} \frac{2 s\left(s-m_{\chi}^{2}\right)+4 u t s / m_{\chi}^{2}}{s^{2}} \\
& +m_{\widetilde{e}_{R}}^{4}\left[\frac{\left(m_{\chi}^{2}-t\right)(-t)}{\left(t-m_{\widetilde{e}_{R}}^{2}\right)^{2}}+\frac{\left(m_{\chi}^{2}-u\right)(-u)}{\left(u-m_{\widetilde{e}_{R}}^{2}\right)^{2}}\right] \\
& +m_{\chi}^{2} m_{\widetilde{e}_{R}}^{2}\left[\frac{(-2 s t)}{s\left(t-m_{\widetilde{e}_{R}}^{2}\right)}+\frac{(-2 s u)}{s\left(u-m_{\widetilde{e}_{R}}^{2}\right)}\right] \\
& +m_{\widetilde{e}_{L}}^{4}\left[\frac{\left(m_{\chi}^{2}-t\right)(-t)}{\left(t-m_{\widetilde{e}_{L}}^{2}\right)^{2}}+\frac{\left(m_{\chi}^{2}-u\right)(-u)}{\left(u-m_{\widetilde{e}_{L}}^{2}\right)^{2}}\right] \\
& +m_{\chi}^{2} m_{\widetilde{e}_{L}}^{2}\left[\frac{(-2 s t)}{s\left(t-m_{\widetilde{e}_{L}}^{2}\right)}+\frac{(-2 s u)}{s\left(u-m_{\widetilde{e}_{L}}^{2}\right)}\right] .
\end{aligned}
$$

Despite the seemingly different appearances of $F_{\text {eff }}^{\tilde{\gamma}}$ and $F^{\tilde{\gamma}}$, it can be verified (at least numerically) that they give identical results. Using $F_{\text {eff }}^{\widetilde{\gamma}}$ it is immediately apparent that the cross section vanishes in the unbroken supersymmetry limit (i.e., $m_{\chi}, m_{\widetilde{e}_{R, L}} \rightarrow 0$ ), as it should. [Note also that for a massless photino (a case of interest in the early literature) the $s$-channel diagram does not contribute.] The $\beta^{8}$ threshold behavior is not so apparent this time. One can first note that near threshold $F_{\text {eff }}^{\tilde{\gamma}}$ becomes independent of $m_{\widetilde{e}_{R, L}}$ and depends only on $m_{\chi}^{2}$. A little algebra then shows that indeed, near threshold, $F_{\text {eff }}^{\gamma} \propto\left(s-m_{\chi}^{2}\right)^{3}$ and thus the same $\beta^{8}$ threshold behavior results, although this time as a result of a cancellation among all of the contributing amplitudes.

The $F_{\text {eff }}^{\tilde{\gamma}}$ form is also useful in exhibiting the dependence of the cross section on the selectron masses. As is evident from Eq. (28), the cross section increases with increasing selectron masses, eventually saturating for very large values of $m_{\tilde{e}}$. Thus, the decoupling theorem still holds (i.e., large values of the sparticle masses have no effect), although its specific implementation here is rather peculiar.

Before moving on to numerical evaluations of the cross sections, let us note that the above expressions for the photino cross section (using either the full or effective couplings) can be adapted very easily to describe gluinogravitino production in quark-antiquark collisions at the Tevatron or CERN Large Hadron Collider (LHC) $(q \bar{q} \rightarrow \widetilde{g} \widetilde{G})$. In this case the process is mediated by $s$-channel gluon exchange and $t$-channel $\widetilde{q}_{L, R}$ exchange. One needs to replace the $e-\widetilde{e}_{R, L}-\chi\left(X_{R, L}\right)$ couplings in Eqs. (17), (18) by those appropriate for $q-\widetilde{q}_{R, L}-\chi$, one needs to replace 


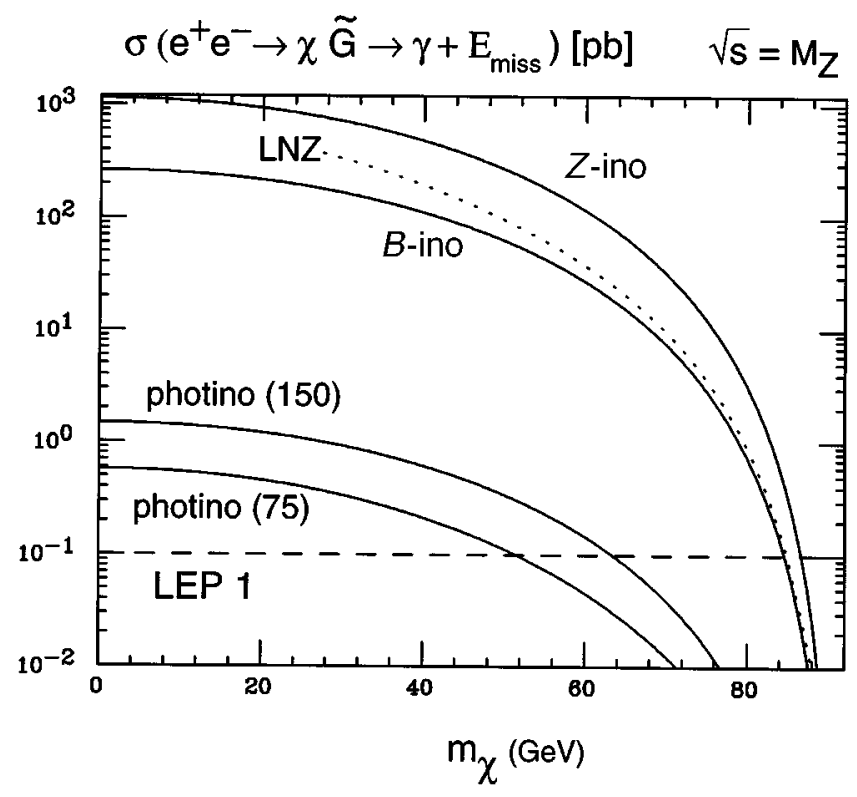

FIG. 2. Single-photon cross sections (in pb) from neutralinogravitino production at LEP 1 versus the neutralino mass $\left(m_{\chi}\right)$ for $m_{\widetilde{G}}=10^{-5} \mathrm{eV}$ and various neutralino compositions. The "photino" curves depend on the selectron mass $(75,150)$. The cross sections scale like $\sigma \propto m_{\tilde{G}}^{-2}$. The dashed line represents the estimated LEP 1 upper limit. Also shown is the result for a one-parameter no-scale supergravity model (LNZ).

the $e-e-\gamma$ coupling $\left[e^{2}\right.$ in Eq. (24)] by the strong coupling $\left(g_{s}^{2}\right)$, and one needs to insert the appropriate color factor. Of course the integration over parton distribution functions also needs to be implemented. (A realistic calculation would also include the gluon-fusion channel, which becomes quite relevant at LHC energies.)

\section{EXPERIMENTAL CONSTRAINTS}

\section{A. LEP 1}

The single-photon signal $\left(\gamma+E_{\text {miss }}\right)$ has been searched for at LEP 1 by various LEP Collaborations [21]. We estimate an upper bound of $0.1 \mathrm{pb}$ on this cross section. This estimate is an amalgamation of individual experimental limits with partial LEP 1 luminosities $\left(\sim 100 \mathrm{pb}^{-1}\right)$ and angular acceptance restrictions $\left(\left|\cos \theta_{\gamma}\right|<0.7\right)$. Note that the singlephoton background at the $Z$ peak (mostly from $\left.e^{+} e^{-} \rightarrow \nu \bar{\nu} \gamma\right)$ is quite significant, as otherwise one would naively expect upper bounds of order $3 / \mathcal{L}<0.03 \mathrm{pb}$. To be conservative, in what follows we apply the $0.1 \mathrm{pb}$ upper limit to our uncut theoretical cross sections.

A numerical evaluation of the single-photon cross section at LEP 1 versus the neutralino mass for $m_{\tilde{G}}=10^{-5} \mathrm{eV}$ is shown in Fig. 2, for different choices of neutralino composition (" $Z$-ino:' $N_{12}^{\prime} \approx 1$, “ $B$-ino:'" $N_{11}=1$, and "photino:'" $\left.N_{11}^{\prime}=1\right)$, and where we have assumed the typical result $B(\chi \rightarrow \gamma \widetilde{G})=1{ }^{4}$ We should remark that our emphasis on

\footnotetext{
${ }^{4}$ Note that this implies a nonvanishing (possibly small) photino component of the neutralino, as would be required in the " $Z$-ino", case discussed above.
}

gauginolike neutralinos is motivated by the explanation of the CDF event, which would become rather unnatural for Higgsino-like neutralinos with a very small gaugino admixture. (Otherwise possible Higgsino admixtures weaken the single-photon signal studied here.) In the photino case the $Z$-exchange amplitude is absent $\left(N_{11}^{\prime}=1 \Rightarrow N_{12}^{\prime}=0\right)$ and one must also specify the selectron masses which mediate the $t$ - and $u$-channel diagrams; we have taken the representative values $m_{\widetilde{e}_{R}}=m_{\widetilde{e}_{L}}=75,150 \mathrm{GeV}$. Increasing the selectron masses further leads to only a small increase in the cross section, e.g., at $m_{\chi}=0$ one finds $\sigma_{\gamma}^{M_{Z}}=1.48,2.09,2.36 \mathrm{pb}$ for $m_{\tilde{e}}=150,300,1000 \mathrm{GeV}$, signalling the reaching of the decoupling limit for large selectron masses discussed in Sec. II B.

In Fig. 2 we also show (dotted line Lopez-NanopoulosZichichi (LNZ)] the results for a well-motivated oneparameter no-scale supergravity model $[8,19]$, which realizes the light gravitino scenario that we study here. In this model the neutralino is mostly gaugino, but has a small Higgsino component at low values of $m_{\chi}$, which disappears with increasing neutralino masses; the neutralino approaches a pure $B$-ino at high neutralino masses. The selectron masses also vary (increase) continuously with the neutralino mass and are not degenerate (i.e., $m_{\widetilde{e}_{L}} \sim 1.5 m_{\tilde{e}_{R}} \sim 2 m_{\chi}$ ).

This figure makes apparent the constraint on the gravitino mass that arises from LEP 1 searches: in some regions of parameter space one must require $m_{G} \gtrsim 10^{-3} \mathrm{eV}$ if $m_{\chi}<M_{Z}$. To make this result more evident, in Fig. 3 we display the lower bound on the gravitino mass versus the neutralino mass that results from the imposition of our estimated upper bound $\sigma_{\gamma}^{M_{Z}}<0.1 \mathrm{pb}$. (The curves that extend beyond $m_{\chi}=M_{Z}$ result from constraints from LEP 2 data and are discussed below.) Note the dependence on the selectron mass in the pure photino case.

\section{B. LEP 161}

Recent runs of LEP at higher center-of-mass energies have so far yielded no excess of single photons over standard model expectations. The latest searches at $\sqrt{s}=161 \mathrm{GeV}$ have produced upper limits on the single-photon cross section $\sigma_{\gamma}^{161} \lesssim 1 \mathrm{pb}[22]$. We have evaluated the single-photon cross sections for the neutralino compositions used in Fig. 2 at $\sqrt{s}=161 \mathrm{GeV}$. This time all cases depend on the choice of selectron masses. The numerical results are shown in Fig. 4, with the experimental upper bound denoted by the dashed line. Note that this line extends only for $m_{\chi}>M_{Z}$, as for $m_{\chi}<M_{Z}$, the much stronger limits discussed in the previous section apply. Moreover, for $m_{\widetilde{G}}=10^{-5} \mathrm{eV}$ (as used in Fig. 4), LEP 1 limits require $m_{\chi} \gtrsim M_{Z}$. As the figure makes evident, for $m_{\chi}<M_{Z}$ the sensitivity to single-photon signals at LEP 2 is not competitive with that at LEP 1.

As discussed above, the cross sections in Fig. 4 increase with increasing selectron masses (saturating at values somewhat larger than the ones shown), and conversely decrease with decreasing selectron masses. The choice of selectron masses also affects the near-threshold behavior of the cross section, with light selectron masses "delaying" the onset of the $\beta^{8}$ threshold dependence (see Fig. 4). Note also that the photino, $B$-ino, and $Z$-ino cross sections become comparable 
Lower limit on $m_{G}(e V)$
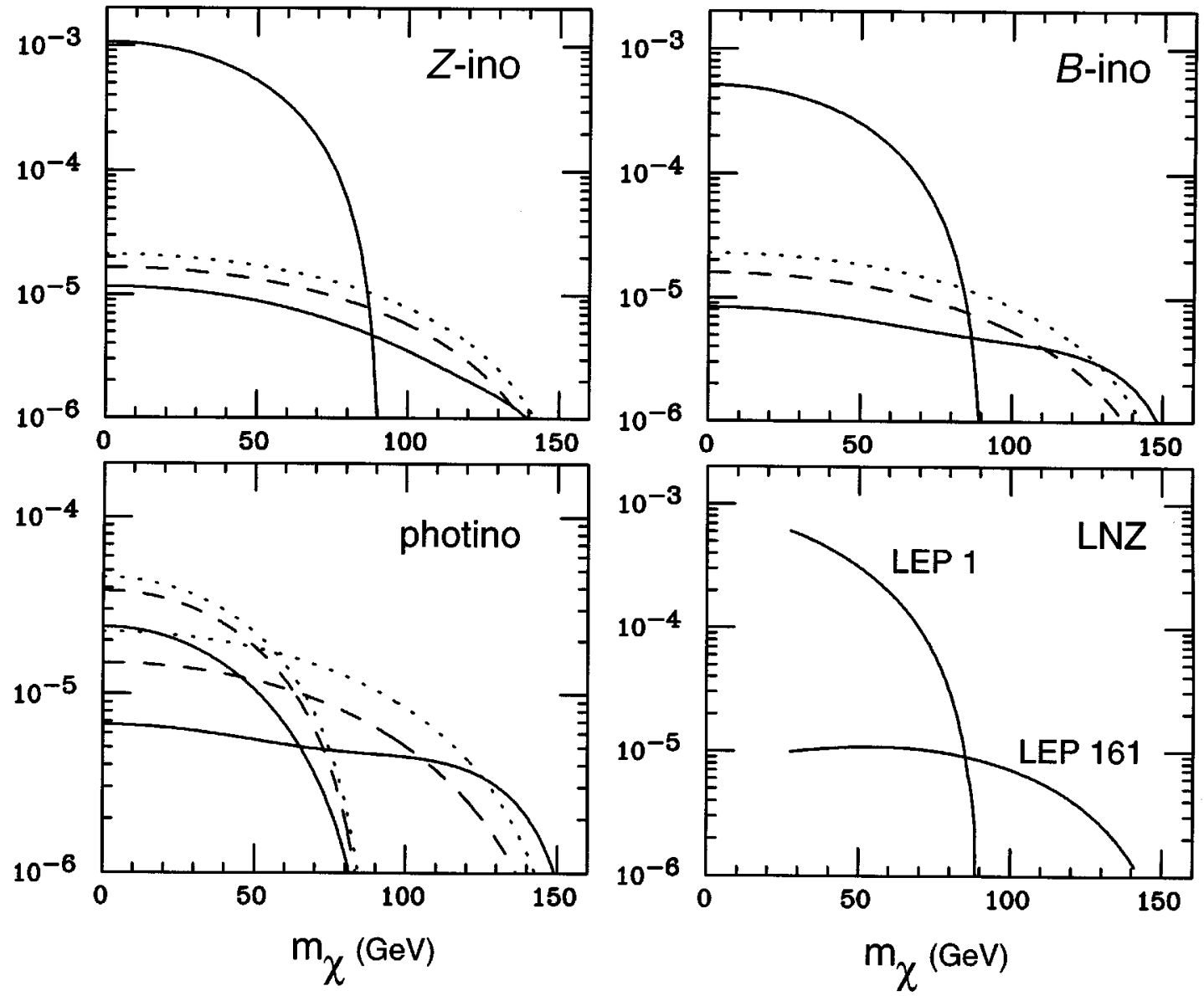

FIG. 3. Lower bounds on the gravitino mass (in eV) as a function of the neutralino mass $\left(m_{\chi}\right)$ that result from single-photon searches $\left(\gamma+\mathrm{E}_{\text {miss }}\right)$ at LEP 1 and LEP 161. In the "photino" case at LEP 1 and the "photino," " $Z$-ino," and " $B$-ino" cases at LEP 161 , the selectron mass influences the results. We have chosen $m_{\tilde{e}}=75,150,300 \mathrm{GeV}$, denoted by solid, dashed, and dotted lines, respectively. Also shown are the bounds in a one-parameter no-scale supergravity model (LNZ).

above the $Z$ pole, when the $Z$-exchange diagram becomes comparable to the other diagrams. In the case of the oneparameter model (LNZ) a peculiar bump appears. This bump is understood in terms of the selectron masses that vary continously with the neutralino mass: at low values of $m_{\chi}$ the selectron masses are light and the cross section approaches the light fixed-selectron mass curves (75); at larger values of $m_{\chi}$ the selectron masses are large and the cross section approaches (and exceeds) the heavy fixed-selectron mass curves (150). This example brings to light some of the subtle features that might arise in realistic models of low-energy supersymmetry.

In spite of their apparent weakeness, LEP 161 limits on the single-photon cross section are useful in constraining the gravitino mass in a neutralino-mass range inaccessible at LEP 1. Indeed, decreasing the gravitino mass in Fig. 4 by a factor of 3 will make the cross sections some ten-times larger. The resulting lower bounds on the gravitino mass from LEP 161 searches are shown in Fig. 3. This figure shows that, as expected, LEP 1 limits dominate for $m_{X} \leq M_{Z}$. However, because of the $\beta^{8}$ threshold behavior at $\sqrt{s}=M_{Z}$, LEP 161 limits "take over" for neutralino masses slightly below $M_{Z}$, and in the "photino" case, considerably below $M_{Z}$.

\section{Single photons versus diphotons}

It has been made apparent in Fig. 3 that for $m_{\chi} \leq M_{Z}$ the gravitino mass is constrained to $m_{\vec{G}} \gg 10^{-5} \mathrm{eV}$. If this was indeed an absolute requirement on the gravitino mass (i.e., for all values of $m_{\chi}$ ) then the cross section for neutralinogravitino production at LEP 2 would be highly suppressed: Fig. 4 shows that $\sigma_{\gamma}^{161} \lesssim 1 \mathrm{pb}$ for $m_{\widetilde{G}}=10^{-5} \mathrm{eV}$ and $\sigma_{\gamma}$ $\propto m_{\widetilde{G}}^{-2}$. In other words, if the minimum observable singlephoton cross section at LEP 2 is $\sim 0.1 \mathrm{pb}$ (i.e., for $\mathcal{L} \sim 100 \mathrm{pb}^{-1}$ ), then $m_{\widetilde{G}} \gtrsim 3 \times 10^{-5} \mathrm{eV}$ appears to be the limit of the sensitivity of LEP 2 .

On the other hand, the process $e^{+} e^{-} \rightarrow \chi \chi \rightarrow \gamma \gamma+E_{\text {miss }}$ is sensitive to $m_{\chi}<\frac{1}{2} \sqrt{s}$ and is independent of the gravitino mass. In light of the single-photon constraints on the gravitino mass obtained above, the diphoton process may be observable at LEP 2 (i.e., $m_{\chi}<\frac{1}{2} \sqrt{s} \lesssim M_{Z}$ ) only if $m_{\widetilde{G}} \gg 10^{-5} \mathrm{eV}$ and therefore single photons will not be simultaneously observable at LEP 2 . Conversely, single photons may be observable at LEP 2 only if $m_{\chi}>M_{Z}$, in which case diphotons may not be observed simultaneously (as they require $\sqrt{s}>2 M_{Z} \approx 190 \mathrm{GeV}$ ). This dichotomy between single-photon and diphoton signals at LEP was first presented in Ref. [13]. 


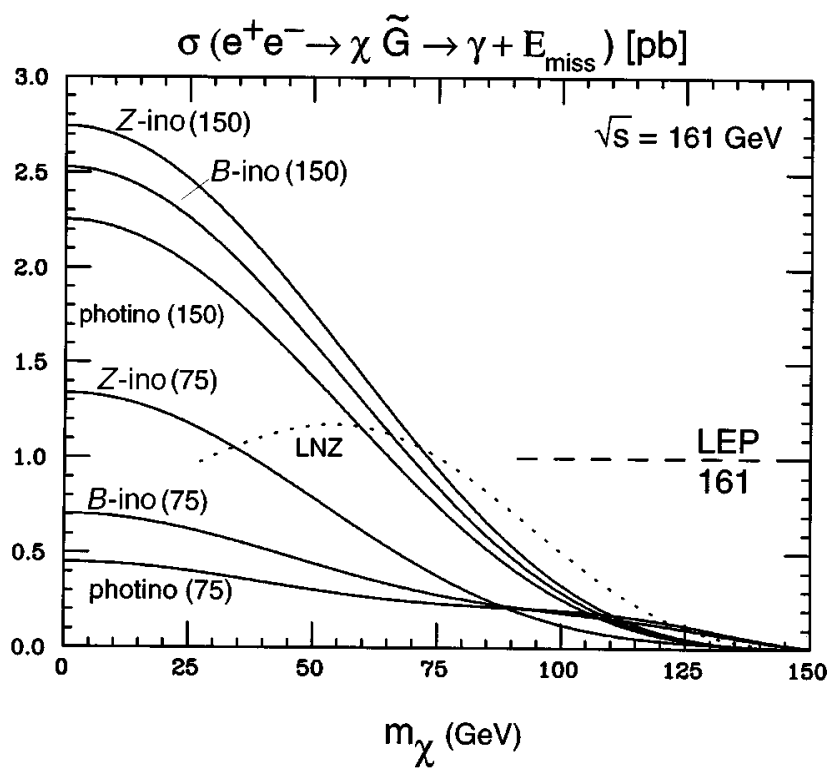

FIG. 4. Single-photon cross sections (in pb) from neutralinogravitino production at LEP 161 versus the neutralino mass $\left(m_{\chi}\right)$ for $m_{\tilde{G}}=10^{-5} \mathrm{eV}$ and various neutralino compositions. The solid curves have a fixed value for the selectron mass $(75,150)$, whereas the dotted curve corresponds to a one-parameter no-scale supergravity model where the selectron masses vary continuously with the neutralino mass. The cross sections scale like $\sigma \propto m_{\tilde{G}}^{-2}$. The preliminary LEP 161 upper limit is indicated.

\section{Other limits}

The above lower limits on $m_{\widetilde{G}}$ are rather significant and improve considerably on previous limits from collider experiments [3-5,21] and astrophysical considerations [12], as long as $m_{\chi}<M_{Z}$. There has also been a recent reassessment [23] of the hadron collider limits obtained via associated gluino-gravitino production $(p \bar{p} \rightarrow \widetilde{g} \widetilde{G})$, and via indirect gluino pair production $(p \bar{p} \rightarrow \widetilde{g} \widetilde{g})$ where in addition to the usual supersymmetry QCD diagrams the gravitino is exchanged in the $t$ and $u$ channels. The multijet signature of these processes has been contrasted with experimental limits from the most recent Tevatron run to show that if gluino pair production is accessible at the Tevatron (i.e., $m_{\tilde{g}} \lesssim 200 \mathrm{GeV}$ ) then a lower limit of $m_{\widetilde{G}}>3 \times 10^{-3} \mathrm{eV}$ results. This limit and our limits in Fig. 3 may be compared by relating the gluino and neutralino masses, as occurs in supergravity models with universal gaugino masses at the unification scale $m_{\tilde{g}} \sim 3 m_{\chi^{ \pm}} \sim 6 m_{\chi}$. Therefore, $m_{\tilde{g}} \lesssim 200 \mathrm{GeV}$ (as required for the bound in Ref. [23] to apply) corresponds to $m_{\chi} \lesssim 35 \mathrm{GeV}$. Consulting Fig. 3, we see that the Tevatron limits are stronger in this neutralino mass range. However, the LEP 1 (161) limit extends up to $m_{\chi} \lesssim M_{Z}\left(2 M_{W}\right)$, which corresponds to $m_{\tilde{g}} \lessgtr 550$ (960) GeV, which is far from the direct reach of the Tevatron.

By considering the further processes $p \bar{p} \rightarrow g S, g P$, where $S$ and $P$ are very light scalar and pseudoscalar particles associated with the gravitino, the lower bound on the gravitino mass becomes much less dependent on the gluino mass and can be taken to be $m_{\widetilde{G}}>3 \times 10^{-4} \mathrm{eV}$ [23]. This lower bound is comparable with those obtained above by considering LEP 1 data. However, this result assumes the existence of addi- tional light and strongly interacting particles $(S$ and $P$ ), an assumption that depends on the detailed nature of the mechanism that leads to a very light gravitino.

\section{E. New channels}

Another set of channels of interest at the Tevatron consist of the associated production of gravitinos with neutralinos or charginos

$$
p \bar{p} \rightarrow \chi \widetilde{G}, \chi^{ \pm} \widetilde{G},
$$

which have the advantage over $p \bar{p} \rightarrow \widetilde{g} \widetilde{G}$ of much less phase space suppression. The most basic channel is $q \bar{q} \rightarrow \chi \widetilde{G}$, which leads to a $\gamma+E_{T \text {,miss }}$ signal. The cross section for this process can be readily obtained from the expressions given in Sec. II by replacing the initial state electron-positron pairs by quark-antiquark pairs, the exchanged selectrons by squarks, and by integrating the resulting expression over parton distribution functions. We have estimated this cross section and find that it may be quite significant: up to $85,25,15 \mathrm{pb}$ for $m_{\chi}=50,75,100 \mathrm{GeV}$ and $m_{\tilde{G}}=10^{-5} \mathrm{eV}$, in favorable regions of parameter space. In the best case scenario of a Tevatron upper limit of $0.1 \mathrm{pb}$ (i.e., 10 events in $\left.\mathcal{L}=100 \mathrm{pb}^{-1}\right)$, one may conclude that $m_{\tilde{G}} \gtrsim(3,1.6,1.2)$ $\times 10^{-4} \mathrm{eV}$ for $m_{\chi}=50,75,100 \mathrm{GeV}$. Taken at face value, these limits are quite competitive with those obtained in Ref. [23]. At the moment there are no single-photon limits available from CDF nor D0.

To improve the visibility of the signal, one may want to consider the $q \bar{q} \rightarrow \chi^{ \pm} \widetilde{G}$ channel which, depending on the chargino decay channel, may lead to $\ell^{ \pm}+\gamma+E_{T \text {, miss }}$ or $2 j+\gamma+E_{T \text {,miss }}$ signals. The leptonic signal appears particularly promising. For all these processes there are some important instrumental backgrounds that need to be overcome. For instance $p \bar{p} \rightarrow W \rightarrow e \nu_{e}$, where the electron is misidentified as a photon (i.e., because of limitations in tracking efficiency), leading to a very large "single-photon" signal $\sigma B\left(W \rightarrow e \nu_{e}\right) \approx 2.4 \times 10^{3} \mathrm{pb}$ [24], which may be reduced significantly by optimizing the tracking efficiency and making suitable kinematical cuts. The other channels mentioned above face similar, although perhaps less severe, instrumental backgrounds ( e.g., $W W \rightarrow e+{ }^{\prime \prime} \gamma$ ' $+E_{T \text {,miss }}$ ).

\section{THE SINGLE-PHOTON SIGNAL}

The total cross section for neutralino-gravitino production has been displayed in Fig. 4 for a specific center-of-mass energy $(\sqrt{s}=161 \mathrm{GeV})$ and for some illustrative choices of parameter values. The analytic expressions given in Sec. II A. allow one to calculate these cross sections for arbitrary values of the parameters. In this section we would like to explore some characteristics of the actual signal, i.e., the energy and angular distributions of the observable photon and the missing invariant mass distribution in the events.

We should note that there is a different kind of singlephoton signal that arises for gravitino masses $\sim 100 \mathrm{eV}$ in neutralino pair production. Because the neutralinos are fairly long lived in this case, only one neutralino may decay to a photon inside the detector [7]. This kind of heavier-gravitino single-photon signal may be distinguished from our present 


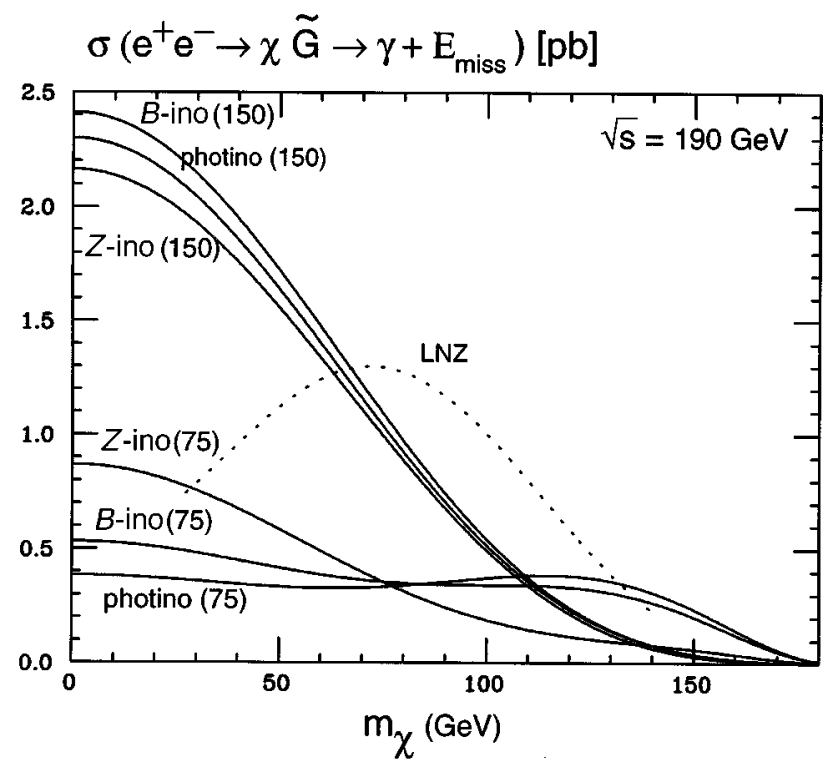

FIG. 5. Single-photon cross sections (in $\mathrm{pb}$ ) from neutralinogravitino production at LEP 190 versus the neutralino mass $\left(m_{\chi}\right)$ for $m_{\tilde{G}}=10^{-5} \mathrm{eV}$ and various neutralino compositions. The solid curves have a fixed value for the selectron mass $(75,150)$, whereas the dotted curve corresponds to a one-parameter no-scale supergravity model where the selectron masses vary continously with the neutralino mass. The cross sections scale like $\sigma \propto m_{\tilde{G}}^{-2}$.

light-gravitino signal by the presence of a visible displaced vertex where the neutralino decayed.

\section{A. Monte Carlo technique}

Our simulation proceeds in a standard way, making use of a "homemade"' Monte Carlo event generator. We start in the rest frame of the decaying neutralino, where we generate $\gamma+\widetilde{G}$ events that are isotropic in this reference frame. Energy-momentum conservation requires $E_{\gamma}^{\prime}=\left|\vec{p}_{\gamma}^{\prime}\right|=\frac{1}{2} m_{\chi}$, which leaves two components of the photon momentum to be generated at random (i.e., $\hat{p}_{\gamma}^{\prime}$ ). We then boost the photon momentum back to the laboratory frame using the neutralino four-momentum $\left(E_{\chi}, \vec{p}_{\chi}\right)$, whose components are constrained by the kinematics of the $e^{+} e^{-} \rightarrow \chi \widetilde{G}$ process:

$$
E_{\chi}=\frac{\sqrt{s}}{2}+\frac{m_{\chi}^{2}}{2 \sqrt{s}}, \quad\left|\vec{p}_{\chi}\right|=\frac{\sqrt{s}}{2}-\frac{m_{\chi}^{2}}{2 \sqrt{s}} .
$$

Here we have two components of the neutralino momentum unconstrained $\left(\cos \theta_{\chi}, \phi_{\chi}\right)$. For fixed values of these angles we obtain $E_{\gamma}$ and $\cos \theta_{\gamma}$ distributions, which are purely kinematical effects. The observable distributions are obtained by varying $\left(\cos \theta_{\chi}, \phi_{\chi}\right)$ and weighing these kinematical distributions with the corresponding dynamical $(1 / \sigma)\left(d \sigma / d \cos \theta_{\chi}\right)$ factors calculable from the expressions given in Sec. II.

In what follows we focus on the case of LEP 190 $(\sqrt{s}=190 \mathrm{GeV})$. First we display in Fig. 5 the total cross sections for single-photon production at this center-of-mass energy. These should give an idea of the reach in neutralino masses that may be accessible at LEP 190. As we expect neutralino-gravitino production to be allowed only for

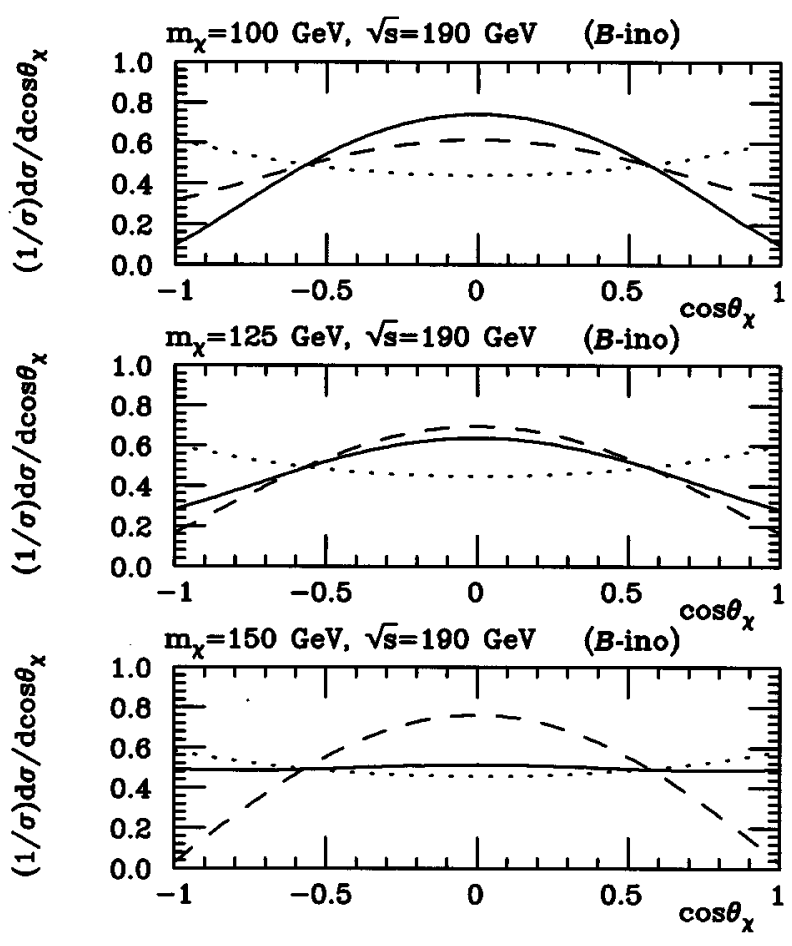

FIG. 6. Normalized angular distribution of neutralinos of $B$-ino composition in neutralino-gravitino production at LEP 190 for $m_{\chi}=100,125,150 \mathrm{GeV}$ and $m_{\tilde{e}}=75$ (solid), 150 (dashed), 300 (dots) $\mathrm{GeV}$

$m_{\chi}>M_{Z}$ (to avoid the stringent LEP 1 lower limits on $m_{\widetilde{G}}$ ), we concentrate on the following three neutralino mass choices: $m_{\chi}=100,125,150 \mathrm{GeV}$. To gain some insight into the final distributions, we start by displaying the normalized neutralino angular distributions $(1 / \sigma)\left(d \sigma / d \cos \theta_{\chi}\right)$ as a function of $\cos \theta_{\chi}$ for $m_{\tilde{e}}=75,150,300 \mathrm{GeV}$, and $B$-ino (Fig. 6), $Z$-ino (Fig. 7), and photino (Fig. 8) neutralino compositions. The total cross sections for each of the curves can be read off Fig. 5 and for convenience have been tabulated in Table I. As can be seen from Figs. 6, 7, and 8, the angular distribution varies quite a bit with neutralino mass, although mostly for light selectron masses. Note that the angular distributions always remain finite, and generally show a preference for the central region.

\section{B. Energy and angular distributions}

The observable photonic energy and angular distributions can be quite unwieldly once we allow for the many choices of parameters that we have considered above. An examination of all parameter combinations shows that both energy and angular distributions are largely insensitive to the neutralino composition, being much more sensitive to the mass parameters (i.e., $m_{\chi}, m_{\tilde{e}}$ ). This result is perhaps not surprising as the observable distributions of relativistic particles are dominated by kinematical effects which depend crucially on the mass parameters. Thus, for brevity we show only the result in the $B$-ino case which, in any event, is representative of typical supergravity models. The energy $\left(E_{\gamma}\right)$ and angular $\left(\cos \theta_{\gamma}\right.$ ) distributions for $m_{\chi}=100,125,150 \mathrm{GeV}$ are shown in Fig. 9 and Fig. 10, respectively, for $m_{\tilde{e}}=75,150,300 \mathrm{GeV}$. These distributions are obtained by 


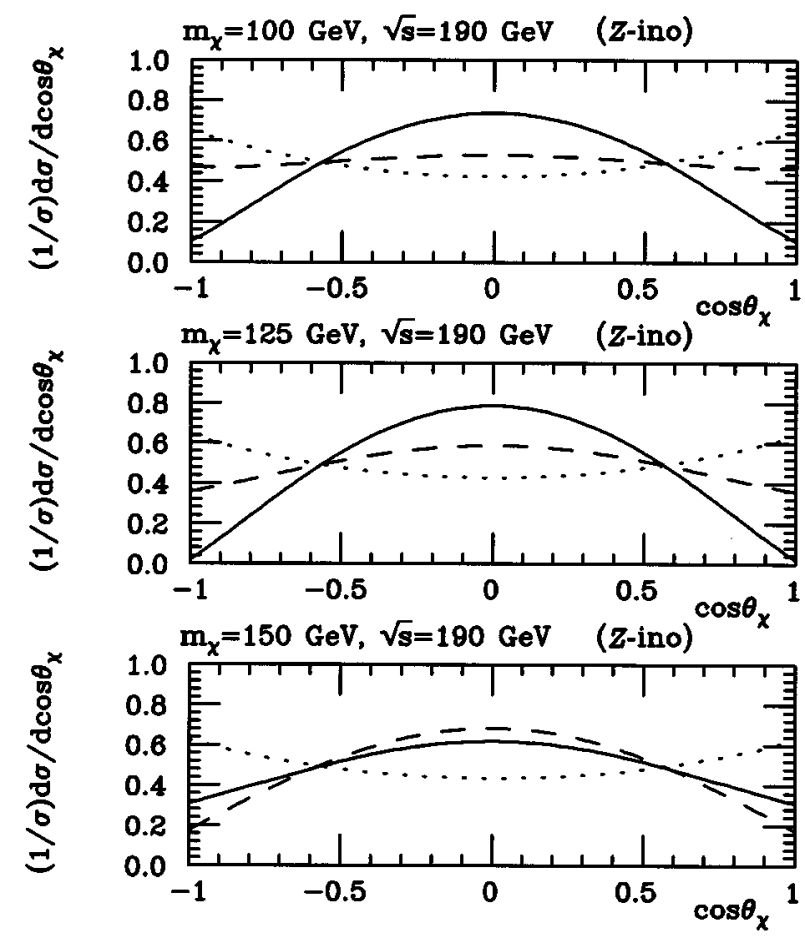

FIG. 7. Normalized angular distribution of neutralinos of $Z$-ino composition in neutralino-gravitino production at LEP 190 for $m_{\chi}=100,125,150 \mathrm{GeV}$ and $m_{\tilde{e}}=75$ (solid), 150 (dashed), 300 (dots) $\mathrm{GeV}$.

generating a total of $100 \mathrm{~K}$ events, which are then binned in $2 \mathrm{GeV} E_{\gamma}$ bins (Fig. 9) and $0.1 \cos \theta_{\gamma}$ bins (Fig. 10). For simplicity, the histograms have been scaled by a factor of 0.01 , thus roughly corresponding to $1 \mathrm{~K}$ generated events.

The energy distributions (Fig. 9) show a significant $m_{\chi}$ and $m_{\tilde{e}}$ dependence. As the neutralino mass grows, it tends to produce harder photons. In fact, it is not hard to show that in the decay $\chi \rightarrow \gamma \widetilde{G}$, with a neutralino energy and momentum as in Eq. (30), the photon energy is restricted to the interval

$$
\frac{m_{\chi}^{2}}{2 \sqrt{s}}<E_{\gamma}<\frac{\sqrt{s}}{2}
$$

TABLE I. Total cross sections corresponding to the differential cross sections shown in Figs. 6,7,8 at LEP 190. All masses in GeV, all cross sections in $\mathrm{pb}$.

\begin{tabular}{lcccc}
\hline \hline Composition & $m_{\chi}$ & $m_{\tilde{e}}=75$ & $m_{\tilde{e}}=150$ & $m_{\tilde{e}}=300$ \\
\hline$B$-ino & 100 & 0.34 & 0.54 & 1.61 \\
& 125 & 0.32 & 0.19 & 0.60 \\
& 150 & 0.20 & 0.04 & 0.11 \\
$Z$ Z-ino & 100 & 0.19 & 0.49 & 1.12 \\
& 125 & 0.10 & 0.17 & 0.42 \\
& 150 & 0.06 & 0.03 & 0.08 \\
Photino & 100 & 0.37 & 0.52 & 1.64 \\
& 125 & 0.37 & 0.18 & 0.61 \\
& 150 & 0.23 & 0.04 & 0.12 \\
\hline \hline
\end{tabular}

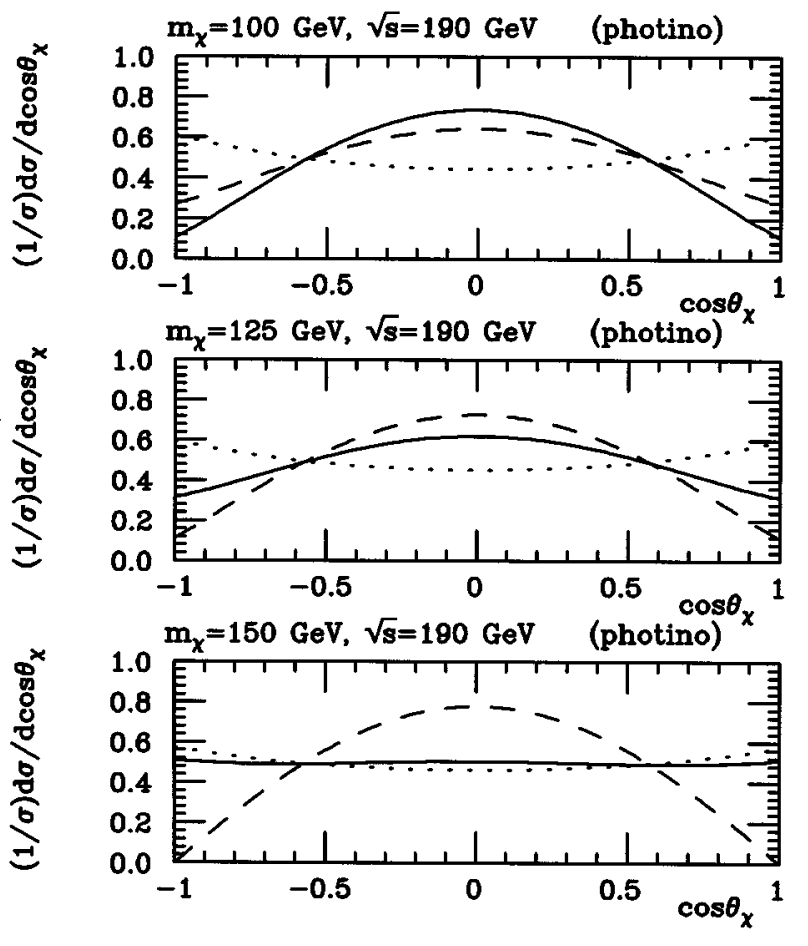

FIG. 8. Normalized angular distribution of neutralinos of photino composition in neutralino-gravitino production at LEP 190 for $m_{\chi}=100,125,150 \mathrm{GeV} \quad$ and $\quad m_{\widetilde{e}}=75$ (solid), 150 (dashed), 300 (dots) $\mathrm{GeV}$.

as faithfully reproduced in the simulations. [Near threshold $\left(m_{\chi} \approx \sqrt{s}\right)$ the photon carries away half of the center-of-mass energy.] These distributions show that any given singlephoton candidate energy $\left(E_{\gamma}\right)$ implies an upper bound on the possible neutralino masses consistent with the candidate event,

$$
m_{\chi}<\sqrt{2 \sqrt{s} E_{\gamma}}
$$

The photonic angular distributions (Fig. 10) are peaked in the forward and backward directions, even more so as the neutralino becomes heavier. The selectron mass has an interesting effect. In the case of $m_{\chi}=100 \mathrm{GeV}$, from Fig. 6 we see that for heavy selectron masses the neutralino angular distribution is fairly flat, and therefore the photonic distributions should reflect only kinematical effects, as they do (i.e., peaked in the forward and backward directions). For light selectron masses, the neutralino avoids the forward and backward directions, and the kinematical effect on the photons is diminished.

\section{Missing mass distribution}

The dominant background to the neutralino-gravitino signal is a single radiative return to the $Z: e^{+} e^{-} \rightarrow \gamma Z \rightarrow \gamma \nu \bar{\nu}$, where the photon is radiated off the initial state and the $Z$ boson tends to be on shell. The most distinctive signature for this background process appears in the missing invariant mass $M_{\text {miss }}=\sqrt{E_{\text {miss }}^{2}-p_{\text {miss }}^{2}}$ distribution, which is strongly peaked at $M_{\text {miss }} \approx M_{Z}$. 

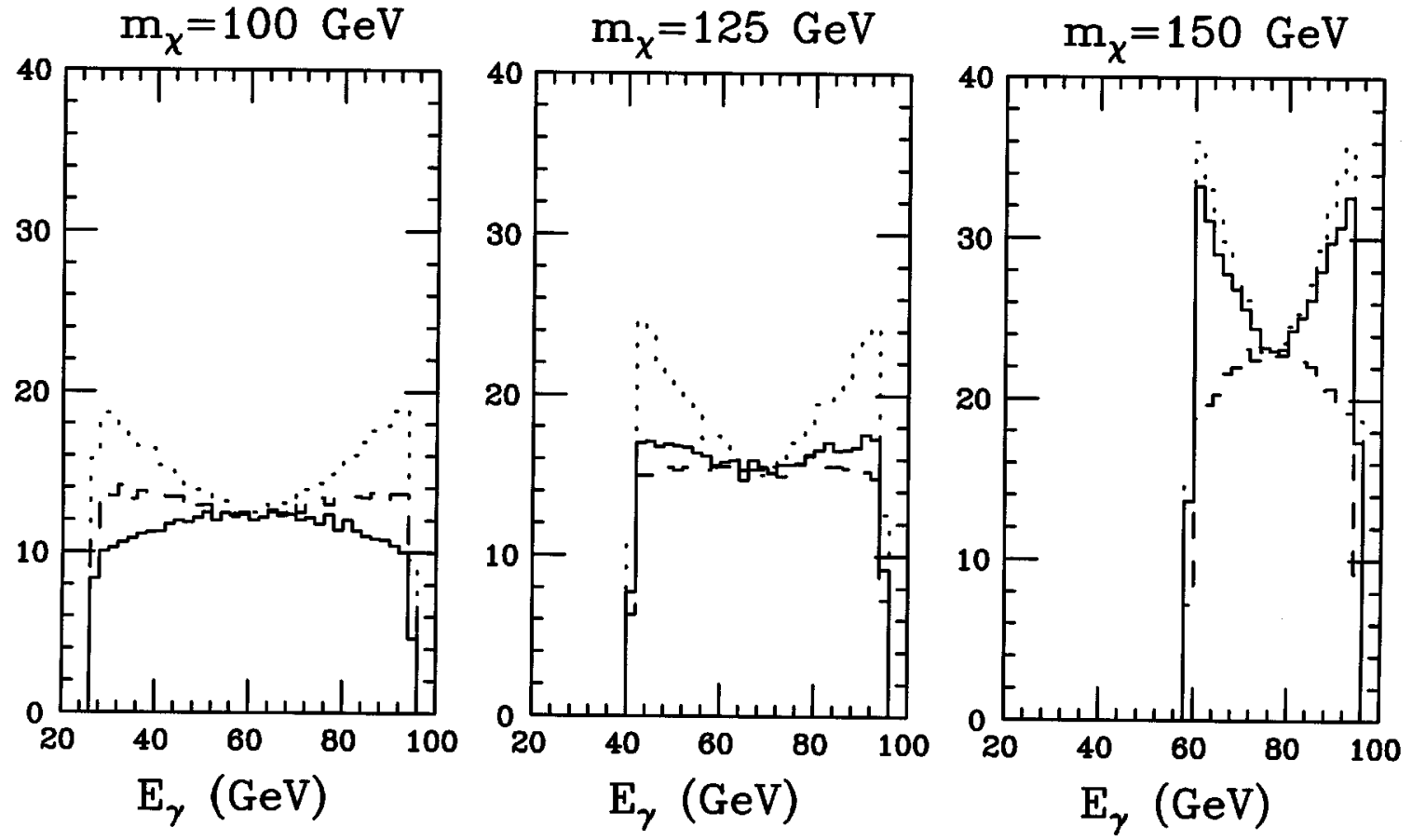

FIG. 9. Photonic energy distributions in neutralino- ( $B$-ino-) gravitino production at LEP 190 for $m_{\chi}=100,125,150 \mathrm{GeV}$ and $m_{\tilde{e}}=75$ (solid), 150 (dashed), and 300 (dots) GeV. (Corresponds to $1 \mathrm{~K}$ events binned in $2 \mathrm{GeV} E \gamma$ bins.)

The missing mass distribution for the signal can be easily determined, as in this case the missing energy and missing momentum are given by

$$
E_{\text {miss }}=\sqrt{s}-E_{\gamma}, \quad p_{\text {miss }}=\left|-\vec{p}_{\gamma}\right|=E_{\gamma},
$$

$$
M_{\text {miss }}=\sqrt{\sqrt{s}\left(\sqrt{s}-2 E_{\gamma}\right)} .
$$

The allowed range of $M_{\text {miss }}$ is obtained by inserting the range of photonic energies in Eq. (31); we obtain

and therefore

$$
0<M_{\text {miss }}<\sqrt{s-m_{\chi}^{2}}
$$
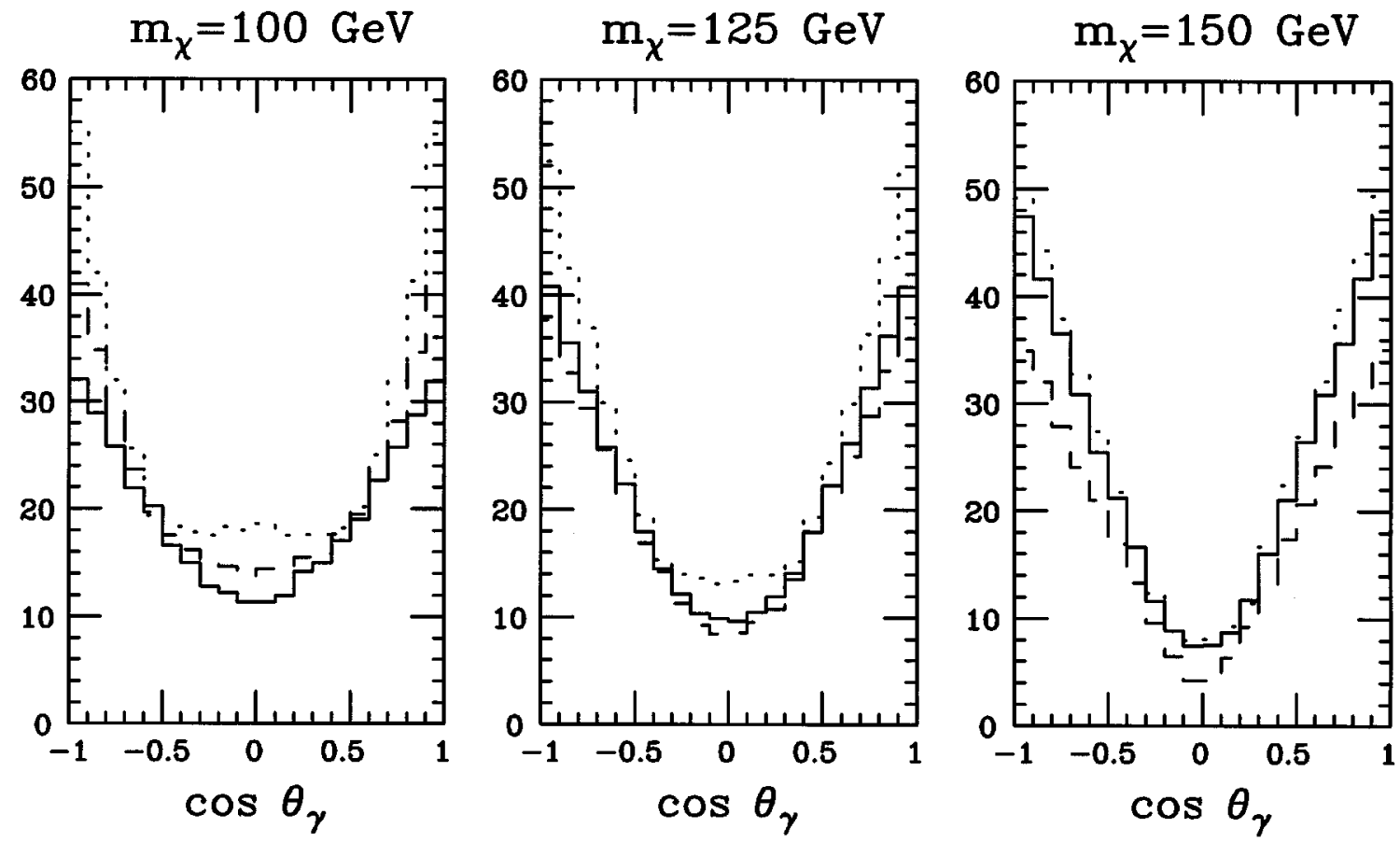

FIG. 10. Photonic angular distributions in neutralino- ( $B$-ino-)gravitino production at LEP 190 for $m_{\chi}=100,125,150 \mathrm{GeV}$ and $m_{\tilde{e}}=75$ (solid), 150 (dashed), and 300 (dots) GeV. (Corresponds to $1 \mathrm{~K}$ events binned in $0.1 \cos \theta_{\gamma}$ bins.) 
Histograms showing missing mass distributions fall in the range specified by Eq. (35) and otherwise favor the upper end of the $M_{\text {miss }}$ range (corresponding to the lower end of the photonic energy range). For brevity, we display these distributions only in the one-parameter model example discussed in Sec. V below.

We note in passing that the complementary diphoton events from neutralino pair production have a dominant background $\left(e^{+} e^{-} \rightarrow \gamma \gamma Z \rightarrow \gamma \gamma \nu \bar{\nu}\right)$ that also peaks for $M_{\text {miss }} \approx M_{Z}[7]$. In this case the missing mass in the diphoton signal varies from zero up to a maximum value of $\frac{1}{2}\left(\sqrt{s}+\sqrt{s-4 m_{\chi}^{2}}\right)$, in contrast with the result for the singlephoton signal in Eq. (35). The $M_{\text {miss }}$ distributions of the single-photon and diphoton signals differ not only in range, but also in shape.

\section{ONE-PARAMETER MODEL EXAMPLE}

It should have become clear from the discussion in Sec. IV, that the signals to be searched for experimentally can have a wide range of characteristics because of the variations in the underlying parameters describing the neutralinogravitino process. In reality, the model of supersymmetry that describes nature will have all its mass parameters correlated in some way, and the actual observations may be a bewildering composite of the many curves shown above. To exemplify this situation, in this section we specialize our results to the case of the one-parameter no-scale supergravity model that has been mentioned at various places in the preceding discussion.

The motivation, construction, and experimental consequences of this model have been expounded on in detail elsewhere $[19,8]$. Perhaps here it would be fit to just mention that from the point of view of unified supergravity models with universal soft-supersymmetry breaking parameters at the unification scale, consistency conditions within the model require that almost all of these parameters vanish (i.e., $m_{0}=A_{0}=B_{0}=0$ ), leaving the universal gaugino mass $\left(m_{1 / 2}\right)$ as the seed of supersymmetry breaking. This choice essentially determines the spectrum of sparticle masses and the various mass relations that have been commented on above. This model also requires that the gravitino be the lightest supersymmetric particle, thus leading to the photonic decay signature for sufficiently light gravitinos.

We have already shown the single-photon cross sections for the LNZ model at LEP 1, LEP 161, and LEP 190 as the dashed lines in Figs. 2, 4, 5, respectively. The cross sections for $\sqrt{s}>M_{Z}$ show a peculiar bump that, as discussed in Sec. III B, can be traced back to the fact that the selectron masses vary with the neutralino mass.

As a first step towards obtaining the angular and energy photonic distributions, in Fig. 11 we show the normalized neutralino angular distributions $(1 / \sigma)\left(d \sigma / d \cos \theta_{\chi}\right)$ for $\sqrt{s}=190 \mathrm{GeV}$ and $m_{\chi}=60,80,100,120,140 \mathrm{GeV}$. The total cross sections in each of these cases are $\sigma=1.2,1.3,1.0,0.6,0.2 \mathrm{pb}$. Note how relatively flat the angular distributions are: no more than a $10 \%$ variation. This is to be contrasted with the wide range of variability observed in the generic cases shown in Figs. 6, 7, and 8. In fact, the results in the one-parameter model resemble those in the generic models when the selectron mass is large (i.e., the dotted

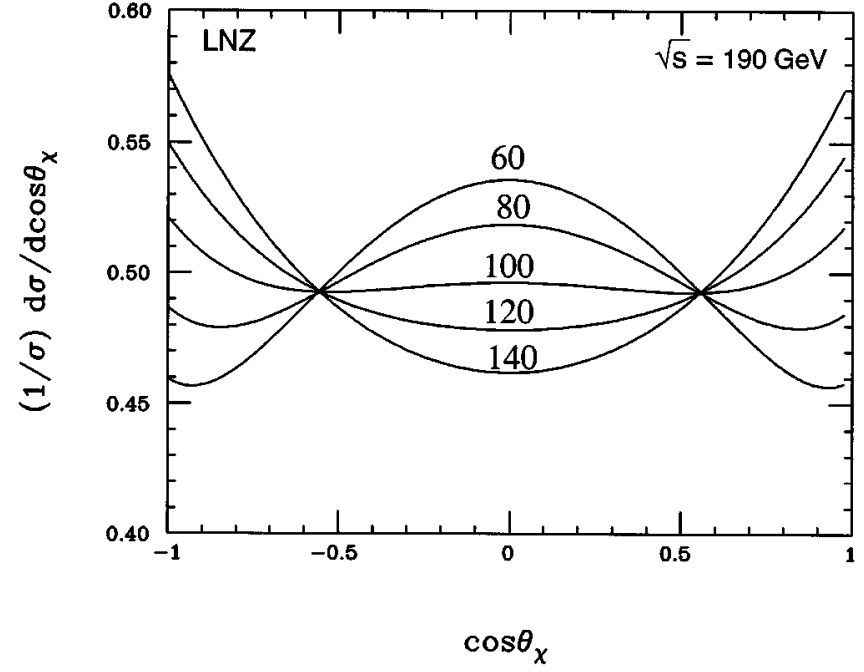

FIG. 11. Normalized angular distribution of neutralinos in neutralino-gravitino production at LEP 190 for $m_{\chi}=60,80,100$, $120,140 \mathrm{GeV}$ in a one-parameter no-scale supergravity model.

lines in those figures, which correspond to $m_{\tilde{e}}=300 \mathrm{GeV}$ ). This is to be expected as in the one-parameter model one has $m_{\widetilde{e}_{L}} \sim 1.5 m_{\widetilde{e}_{R}} \sim 2 m_{\chi}$, indicating increasingly heavier selectrons.

Following the method outlined in Sec. IV, in Figs. 12 and 13 we display the photonic energy and angular distributions at LEP 190 for three representative neutralino masses $\left(m_{\chi}=100,120,140 \mathrm{GeV}\right)$. The energy distributions show the same restrictive photon energy behavior as predicted by Eq. (31). The angular distributions are also peaked in the forward and backward directions.

Finally we consider the missing mass distributions, which are obtained from Eq. (34), and are shown in Fig. 14. We note the range of $M_{\text {miss }}$, as prescribed by Eq. (35), and the tendency to favor missing mass values toward the upper end of the allowed interval. For the neutralino mass choices

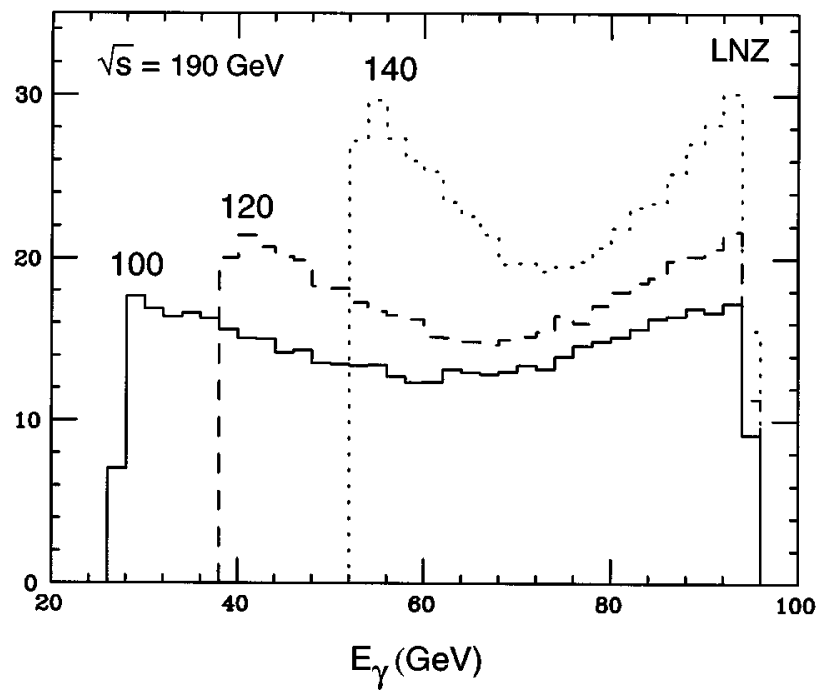

FIG. 12. Photonic energy distributions at LEP 190 in a oneparameter no-scale supergravity model for $m_{\chi}=100,120$, $140 \mathrm{GeV}$. (Corresponds to $1 \mathrm{~K}$ events binned in $2 \mathrm{GeV} E \gamma$ bins.) 


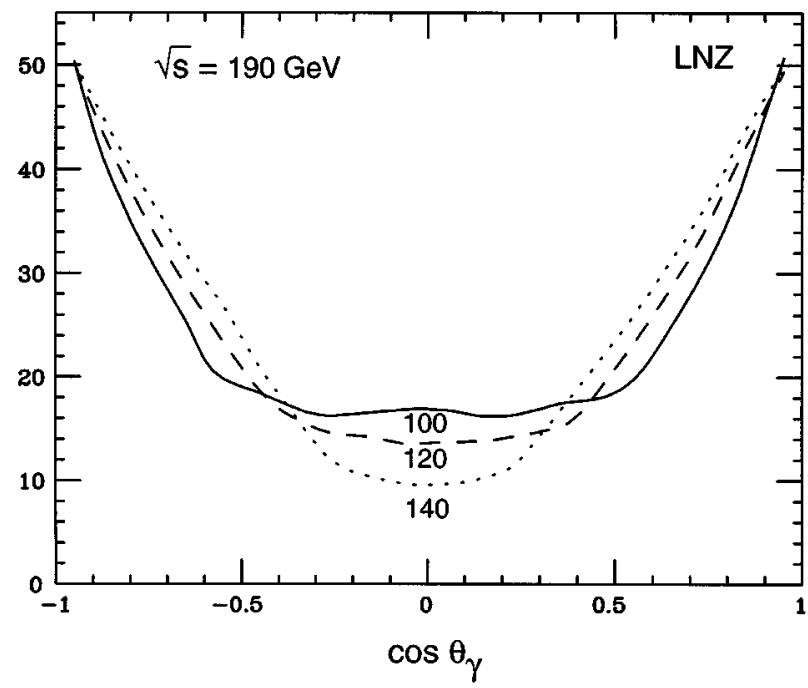

FIG. 13. Photonic angular distributions at LEP 190 in a oneparameter no-scale supergravity model for $m_{\chi}=100,120$, $140 \mathrm{GeV}$.

shown, the missing mass shows a distinct preference to be larger than $M_{Z}$. (This is in contrast with the $M_{\text {miss }}$ distribution in diphoton events, which is more evenly distributed.)

\section{CONCLUSIONS}

In this paper we have attempted to study in some detail the physics of supersymmetric models with a gravitino light enough that it can be produced directly at collider experiments: $m_{\tilde{G}} \lesssim 10^{-3} \mathrm{eV}$ accompanied by neutralinos with nonnegligible gaugino admixture. Our discussion has centered mainly around LEP, from where the strongest constraints can be obtained at the moment. We have nonetheless outlined the corresponding program to be followed at the Tevatron, where instrumental backgrounds make identification of the single-photon signal a more challenging task.

We have provided new and explicitly analytical expressions for neutralino-gravitino differential cross sections at $e^{+} e^{-}$colliders and have discussed some of the theoretical

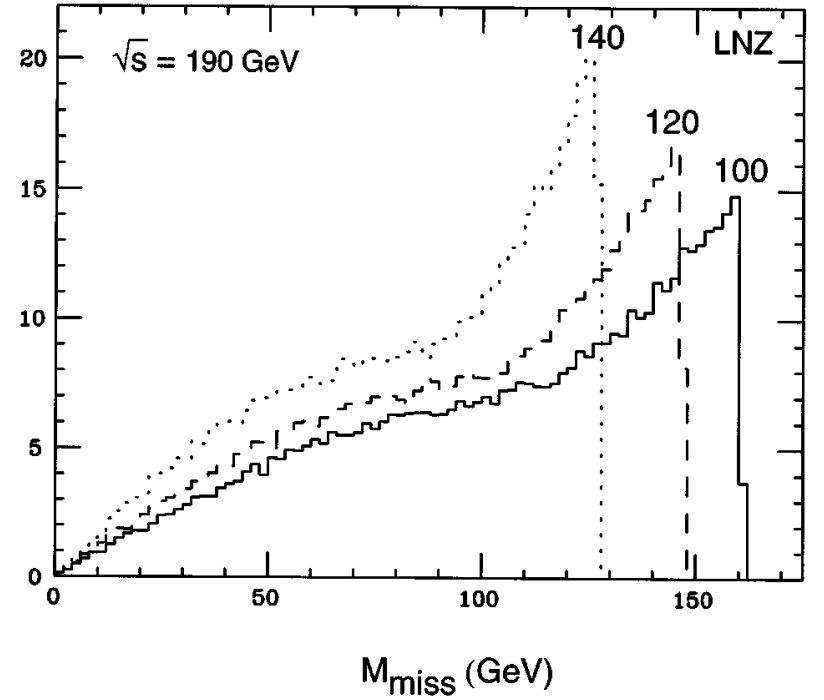

FIG. 14. Missing invariant mass distributions at LEP 190 in a one-parameter no-scale supergravity model for $m_{\chi}=100,120$, $140 \mathrm{GeV}$. (Corresponds to $1 \mathrm{~K}$ events binned in $2 \mathrm{GeV} M_{\text {miss }}$ bins.)

subtleties involved in the calculation and some of the peculiar parameter dependences of the cross section. We have used our expressions to obtain new lower bounds from LEP 1 data on the gravitino mass for $m_{\chi}<M_{Z}$. Weaker limits from LEP 2 are obtained at higher neutralino masses. Our study includes a Monte Carlo simulation of the single-photon signal, which should be helpful in the experimental analyses that are just now getting underway. We have also specialized the results to our one-parameter no-scale supergravity model, where the signals can be analyzed much more simply because of the tight correlations between the model parameters.

\section{ACKNOWLEDGMENTS}

J.L. would like to thank G. Eppley, T. Gherghetta, and T. Moroi for useful discussions. The work of J.L. has been supported in part by U.S. DOE Grant No. DE-FG05-93-ER40717 and that of D.V.N. by U.S. DOE Grant No. DE-FG0591-ER-40633.
[1] P. Fayet, Phys. Lett. 69B, 489 (1977); 70B, 461 (1977); 84B, 421 (1979); 86B, 272 (1979).

[2] J. Ellis, K. Enqvist, and D. Nanopoulos, Phys. Lett. 147B, 99 (1984); see also 151B, 357 (1985).

[3] P. Fayet, Phys. Lett. 175B, 471 (1986).

[4] D. Dicus, S. Nandi, and J. Woodside, Phys. Rev. D 41, 2347 (1990); 43, 2951 (1991).

[5] D. Dicus, S. Nandi, and J. Woodside, Phys. Lett. B 258, 231 (1991).

[6] D. Stump, M. Wiest, and C.-P. Yuan, Phys. Rev. D 54, 1936 (1996); S. Dimopoulos, M. Dine, S. Raby, and S. Thomas, Phys. Rev. Lett. 76, 3494 (1996).

[7] S. Ambrosanio, G. Kane, G. Kribs, S. Martin, and S. Mrenna, Phys. Rev. Lett. 76, 3498 (1996); Phys. Rev. D 54, 5395 (1996).
[8] J. L. Lopez and D. V. Nanopoulos, Mod. Phys. Lett. A 11, 2473 (1996); Phys. Rev. D 55, 4450 (1997).

[9] S. Park in Proceedings of the 10th Topical Workshop on Proton-Antiproton Collider Physics, Fermilab, 1995, AIP Conf. Proc. No. 357, edited by R. Raja and J. Yoh (AIP, New York, 1995), p. 62.

[10] K. Choi, E. Chun, and J. Lee, Report No. hep-ph/9611285 (unpublished).

[11] See, e.g., T. Moroi, Ph.D. thesis, 1995; T. Gherghetta, Nucl. Phys. B485, 25 (1997).

[12] See, e.g., J. Grifolds, R. Mohapatra, and A. Riotto, Report No. hep-ph/9610458 (unpublished); Report No. hep-ph/9612253 (unpublished).

[13] J. L. Lopez, D. V. Nanopoulos, and A. Zichichi, Phys. Rev. Lett. 77, 5168 (1996). 
[14] S. Dimopoulos, S. Thomas, and J. Wells, Phys. Rev. D 54, 3283 (1996); K. Babu, C. Kolda, and F. Wilczek, Phys. Rev. Lett. 77, 3070 (1996).

[15] Joint Particle Physics Seminar and LEPC Open Session, CERN, 1996 (unpublished). Presentations by W. de Boer (DELPHI Collaboration), R. Miquel (ALEPH Collaboration), and M. Pohl (L3 Collaboration) http://hpl3sn02.cern.ch/, N. Watson (OPAL Collaboration) http://www.cern.ch/Opal/.

[16] J. Ellis, J. L. Lopez, and D. V. Nanopoulos, Report No. hep-ph/9610470 (unpublished).

[17] S. Ambrosanio, G. Kane, G. Kribs, S. Martin, and S. Mrenna, Phys. Rev. D 55, 1372 (1997).

[18] J. Hisano, K. Tobe, and T. Yanagida, Phys. Rev. D 55, 411 (1997).
[19] J. L. Lopez, D. V. Nanopoulos, and A. Zichichi, Phys. Rev. D 49, 343 (1994); Int. J. Mod. Phys. A 10, 4241 (1995).

[20] H. Haber and G. Kane, Phys. Rep. 117, 75 (1985).

[21] L3 Collaboration, O. Adriani et al., Phys. Lett. B 297, 469 (1992); OPAL Collaboration, R. Akers et al., Z. Phys. C 65, 47 (1995); DELPHI Collaboration P. Abreu et al., Report No. CERN-PPE/96-03 (unpublished).

[22] W. de Boer, in Joint Particle Physics Seminar and LEPC Open Session, CERN, 1996 [15].

[23] D. Dicus and S. Nandi, Report No. hep-ph/9611312 (unpublished).

[24] D0 Collaboration, S. Abachi et al., Phys. Rev. Lett. 75, 1456 (1995); CDF Collaboration F. Abe et al., ibid. 76, 3070 (1996). 